\title{
Novel exostosin-2 mutation identified in a Chinese family with hereditary multiple osteochondroma
}

\author{
WEIWEI RUAN ${ }^{1}, \mathrm{LI} \mathrm{CAO}^{2}, \mathrm{ZHONGHUA} \mathrm{CHEN}^{2}, \mathrm{MINGXIANG} \mathrm{KONG}^{2}$ and QING BI ${ }^{2}$ \\ ${ }^{1}$ Department of Orthopedics, Tongde Hospital of Zhejiang Provincial, Hangzhou, Zhejiang 310012; \\ ${ }^{2}$ Department of Orthopedics and Joint Surgery, Zhejiang Provincial People's Hospital, Hangzhou, Zhejiang 310014, P.R. China
}

Received September 17, 2015; Accepted December 15, 2017

DOI: $10.3892 / \mathrm{ol} .2018 .7838$

\begin{abstract}
Hereditary multiple osteochondroma (HMO) is an autosomal dominant genetic disorder characterized by multiple outgrowing bony tumors capped by cartilage, generally affecting the metaphyses. The disease is known as hereditary multiple exostoses, familial exostosis, multiple cartilaginous exostoses or hereditary malformation of cartilage. The prevalence of HMO in Europe and the Unites States is $\sim 1: 100,000$, although it has not been reported in China. The disease is often accompanied by pain, asymmetry and skeletal malformations, including forearm and leg bending deformities, limb length discrepancies, and knee internal and external rotation abnormalities. Mutations to exostosin-1 (EXT1) and EXT2 mutations cause insufficient heparan sulfate biosynthesis, leading to chondrocyte proliferation, abnormal bone growth in neighboring regions, multiple exostoses, and ultimately malignant transformation. The risk of malignant degeneration to osteochondrosarcoma increases with age, despite the low lifetime risk $(\sim 1 \%)$. The present study selected a clinical feature of typical HMO pedigrees, and examined mutations in family members by Sanger sequencing. Each of the five patients examined had a novel heterozygous nonsense mutation, c. $67 \mathrm{C}>\mathrm{T}$ p.Arg $23^{*}$. The mutation is located prior to the EXT2 exostosin domains in the amino acid sequence and results in a protein truncation of the $705 \mathrm{C}$-terminal amino acids. The present study provides molecular genetic evidence for a novel causal mechanism of HMO, and provides the basis for clinical genetic counseling for similar diseases.
\end{abstract}

\section{Introduction}

Hereditary multiple osteochondroma (HMO), also known as hereditary multiple exostoses, is characterized by multiple

Correspondence to: Dr Qing Bi, Department of Orthopedics and Joint Surgery, Zhejiang Provincial People's Hospital, 158 Shangtang Road, Hangzhou, Zhejiang 310014, P.R. China E-mail: 13588302991@126.com

Key words: hereditary multiple osteochondroma, exostosin-2, nonsense mutation exogenous exostoses in the skeletal system $(1,2)$. The incidence of HMO in Europe is 1:100,000 (3), and in the United States is $\sim 1: 50,000$ (4); however, to the best of our knowledge, its incidence in China is presently unknown. It occurs more frequently in males than in females $(\sim 1.5: 1)$. Symptoms in females are not serious and the disease is often not diagnosed $(5,6)$. HMO is autosomal dominant (7) and has an penetrance of $100 \%(3,4)$. Approximately $70 \%$ of patients with HMO have a family history of the disorder $(5,8,9)$. The disease affects regions of the humerus (10-50\%), forearm (39-60\%), knee $(33 \%)$, ankle $(25 \%)$, and other long bones; the scapula and ribs are affected, and the majority of osteochondroma is distributed symmetrically (4,10-12).

Studies concerning the etiology and molecular genetic background of HMO have indicated that the majority cases are associated with mutations in the exostosin (EXT) tumor inhibition genes (13); specifically, EXT1, EXT2, and EXT3, which are located at 11p11-13, 8c23-24.1, and on chromosome 19p, respectively, are associated with HMO $(14,15)$. EXT encodes a type II transmembrane protein that is closely associated with the synthesis of heparan sulfate (HS), a key component of cartilage (16). Mutations in the EXT gene affect glycosyltransferase activities, resulting in an HS synthesis barrier. Nonsense mutations, frameshift mutations and splice site mutations account for $80 \%$ of mutations in patients with HMO.

In the present study, the clinical data of members from three generations of a single family with HMO were collected and statistically analyzed. Using two known genes of HMO, EXT1 and EXT2, the genomic DNA of the family members was directly sequenced using a polymerase chain reaction (PCR), in order to identify the pathogenesis of the family with HMO and explore the potential underlying mechanism. The results of the present study may be useful for the clinical genetic counseling of similar diseases in the future.

\section{Materials and methods}

Patients and clinical data. A three-generation HMO family, JX-JJ001 (Jiangxi, China) were the subject of the present study (Fig. 1). Diagnostic criteria: X-ray examination revealed that the long bone metaphysis had at least two or more osteochondromas. According to the above criteria, it was confirmed that five patients ( 4 male, 1 female) in the family exhibited generation inheritance of HMO. All participants provided informed 
consent for specimen and information collection. Clinical data were obtained from pedigree patients by the questionnaire survey, which included family history, age of onset, age of diagnosis, evolution of the disease, history of surgery, the location of exogenous bone warts, limb deformities or limited joint activity, and other relevant clinical symptoms such as malignant transformation and mortality (Fig. 1 and Table I). The five patients within the family and three healthy individuals were involved in the present study. All five HMO patients in the family exhibited multiple exostosis, including in the femur, tibia, fibula and knee. All five patients had shorter than normal limbs, but did not exhibit serious limb deformity, with no symptoms of hip or knee valgus or limited mobility. None of the patients had serious complications, only feeling that the mass was protruding into the body surface or occasional pain (mean visual analogue scale score, 2.7 points); however, these symptoms did not limit the mobility of the knee. The average age of the HMO family was 37.4 years old. Prior to the present study, no patient had undergone surgical resection of osteochondroma. The mean height of the pedigree patients was $155.4 \mathrm{~cm}$ in man, much lower than that of the China National Sports General data (which was $169.9 \mathrm{~cm}$ in men from 35-39 years) released in 2010 by the National Physical Monitoring Bulletin (17).

In addition to members of the JX-JJ001 family, a further 300 volunteers (153 males and 147 females; $19-45$ years old; median age 35 years old) who were healthy and were not related to the family participated in the study. The present study was approved by the Zhejiang Provincial People's Hospital Ethics Committee (Hangzhou, China), and all participants provided written informed consent.

Molecular analysis. The peripheral blood from the pedigree patients and the non-patient members was drawn and added to the EDTA anticoagulant tube, Next, the DNA was extracted from the blood using the AxyPrep Nucleic Acid Purification kit (Axygen Scientific, Inc., Union City, CA, USA) in accordance with the manufacturer's protocol. Following this, a Nanodrop2000 spectrophotometer (Nanodrop Technologies; Thermo Fisher Scientific, Inc., Wilmington, DE, USA) was used to quantify DNA. Primer 5.0 (Premier Biosoft International, Palo Alto, CA, USA) was used to design primers and primer sequences (Table II) for all exons of EXT1 and EXT2 (including the $100 \mathrm{bp}$ base of exon and intron junctions). PCR (KAPA2G Fast Multiplex Mix; Kapa Biosystems, Inc., Wilmington, MA, USA) was used for the expansion of genomic DNA, with thermocycling conditions as follows: $94^{\circ} \mathrm{C}$ for $5 \mathrm{~min}$, followed by $94^{\circ} \mathrm{C}$ for $30 \mathrm{sec}$ and $60^{\circ} \mathrm{C}$ for $30 \mathrm{sec}$, for $30 \mathrm{cycles}$ each, followed by $72^{\circ} \mathrm{C}$ for $1 \mathrm{~min}, 72^{\circ} \mathrm{C}$ for $5 \mathrm{~min}$ and $12^{\circ} \mathrm{C}$ for $10 \mathrm{~min}$. Amplified DNA product was subjected to Sanger sequencing analysis using the BigDye Terminator v3.1 Cycle Sequencing kit (Applied Biosystems; Thermo Fisher Scientific, Inc., Waltham, MA, USA) and an ABI 3730XL automatic sequencer (Applied Biosystems; Thermo Fisher Scientific, Inc.). Samples from the patients and healthy controls were bi-directionally sequenced and the sequencing results were analyzed using Sequencer Demo 3.0 (Gene Codes Corporation, Ann Arbor, MI, USA) and Mutation Surveyor Demo V4.0 software (SoftGenetics, LLC, State College, PA, USA), with National Centre for Biotechnology

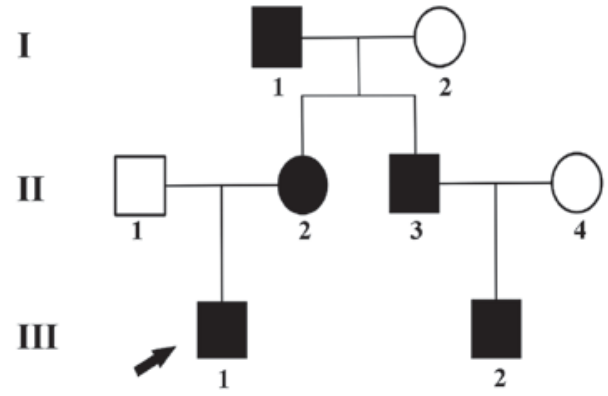

Figure 1. Pedigree of the Chinese family with hereditary multiple exostoses The filled symbols represent affected individuals, and empty symbols indicate unaffected family members. The proband (III1; first member of the third generation of the family) is noted with an arrow. There is no consanguinity in this family. Squares indicate male patients, circles indicate female patients. HMO, hereditary multiple osteochondroma.

Information (EXT1, NM_000127.2; EXT2, NM_001178083.1; https://www.ncbi.nlm.nih.gov/) sequences used as reference genes.

Computer simulation analysis. The present study used Mutation Taster software (18) to predict the pathogenicity of mutations.

Evolutionary conservation and structural prediction. ClustalX 1.83 (19) to analyze the conservativeness of mutant amino acids by comparing the human wild-type $E X T 2$ gene protein sequence (NP_000118) and the EXT2 gene sequence (sequence from www.ensembl.org/) of other species, including mackerel, fruit fly, mouse, Xenopus laevis and Caenorhabditis elegans.

\section{Results}

DNA sequencing identified a novel mutation to the EXT2 gene in all patients with HMO. The tissue structure (Fig. 2) of patients with HMO was characterized by a cartilage cap that was covered by a fibrous perichondrium and merged into an underlying spongy bone. Each exon of the EXT1 and EXT2 genes was sequenced in 5 patients (I1, II2, II3 III1, and III2, as shown in Fig. 1) and 3 normal members (I2, II1 and II4, as shown in Fig. 1) of the HMO family JX-JJ001. Following the data filtering procedures, all of the HMO patients were found to have mutations in the EXT2 gene. A novel nonsense mutation was identified on the EXT2 gene in the five HMO patients: c. $67 \mathrm{C}>\mathrm{C} / \mathrm{T}$ (amino acid R23*), (Fig. 3), it is worth noting that this novel nonsense 23R $>X$ mutation occurred only in the five HMO patients and was not found in other family members. Therefore, we hypothesize that this novel EXT2 mutation site may be a pathogenic mutation site. According to this finding, the DNA of 300 healthy volunteers who were not associated with the family members was sequenced, and it was found that none of the EXT2 genes in the 300 healthy volunteers had a $23 \mathrm{R}>\mathrm{X}$ mutation. Thus, this novel nonsense mutation $23 \mathrm{R}>\mathrm{X}$ in the EXT2 gene is leading to pathogenicity of the HMO family JX-JJ001.

$23 R>X$ mutation had a notable effect on the function of the EXT2 gene. To understand the effect of $23 \mathrm{R}>\mathrm{X}$ on the function of the EXT2 gene, further computer simulation analysis was performed. The protein encoded by the EXT2 
Table I. Clinical data for five hereditary multiple osteochondroma patients.

\begin{tabular}{|c|c|c|c|c|c|}
\hline Patient & Sex & Height, cm & $\begin{array}{c}\text { Age of onset, } \\
\text { years }\end{array}$ & $\begin{array}{l}\text { Age at time of study, } \\
\text { years }\end{array}$ & Localization \\
\hline 1 & Male & 172.5 & 75 & 75 & Femurs, tibia, fibula and knee joints \\
\hline 2 & Female & 157.4 & 37 & 45 & Femurs, tibia, fibula and knee joints \\
\hline 3 & Male & 159.2 & 38 & 42 & Femurs, tibia, fibula and knee joints \\
\hline 4 & Male & 160.5 & 7 & 15 & Femurs, tibia, fibula and knee joints \\
\hline 5 & Male & 129.4 & 6 & 10 & Femurs, tibia, fibula and knee joints \\
\hline
\end{tabular}

Table II. Primer sequences used to sequence the EXT2 genes.

\begin{tabular}{|c|c|c|}
\hline Primers & Forward & Reverse \\
\hline EXT2-E1 & 5'-ATTGCCCTCCAGGAATGTTA-3' & 5'-GCAGGAGTGGAAATCGGAG-3' \\
\hline EXT2-E2 & 5'-GGCGTGGTGGTCACAGTTAC-3' & 5'-ACCAACTCAAGAGCAGAAGCA-3' \\
\hline EXT2-E3 & 5'-CTGTTGGGATTTCCAGGAGTTT-3' & 5'-TGCCAGGACATAAGCCCTAACT-3' \\
\hline EXT2-E4 & 5'-TGATTCAAGGATAGAACGCAG-3' & 5'-AAACAAAGGAGAGAACGGAGT-3' \\
\hline EXT2-E5 & 5'-GTGGAGGTGAAGACTGGTAAGG-3' & 5'-CACAAGACACCAGACATCCAAG-3' \\
\hline EXT2-E6 & 5'-GGCGTCAACCCTTGTAGAAAC-3' & 5'-CCTTGGTTTGTGAACTGCTCT-3' \\
\hline EXT2-E7 & 5'-GAAGGAGGTTTGGGATGTTGTT-3' & 5'-AAGTAAACCCCACTCAGGCATT-3' \\
\hline EXT2-E8 & 5'-AAGTGTGCCTGGTTGGAGTG-3' & 5'-ACTGCTGAAACCCTGCTGTG-3' \\
\hline EXT2-E9 & 5'-TAAAGGAATTAGCCTAACCTGGAG-3' & 5'-CCCAAGTATAAAAAGCACACTCTC-3' \\
\hline EXT2-E10 & 5'-GTAAAAGCCACCAAGCCTGC-3' & 5'-GTATGCCAGGGCTTGGAGTT-3' \\
\hline EXT2-E11 & 5'-CTTTGGATTTGATGAGAGCCG-3' & 5'-CCCACACTCTTACGCACACCT-3' \\
\hline EXT2-E12 & 5'-TCTCCAGAATCCCATTATGACCT-3' & 5'-ATGGTTATCTCGAAGTGACAGGA-3' \\
\hline EXT2-E13 & 5'-GCСТССТTTТАСССТТССТАТT-3' & 5'-GACCGCATCAATCATAGAACCT-3' \\
\hline EXT2-E14 & 5'-CTTGTGAGTTCTGCCGTTGG-3' & 5'-GACCCTTCCAGCCATTACAAA-3' \\
\hline EXT2-E14-CX & & 5'-ATGGAGGTGACTATGGCTAC-3' \\
\hline EXT2-E15-1 & 5'-TTTGCTGTTATCTCTCAACCTCT-3' & 5'-AGGAATTGGTGTTTAAGACACAG-3' \\
\hline EXT2-E15-2 & 5'-TACATCAATGAGTTCTTTCAGGGA-3' & 5'-ACGCTGACTGGCAAAACAACTA-3' \\
\hline EXT2-E15-CX & & 5'-AACTGTGGCTAATCTTAATA-3' \\
\hline
\end{tabular}

EXT2-E1, exostosin-2 exon 1.

gene was composed of 728 amino acids and that it had two notable domains: The exostosin domain (between the 101 and 380 amino acids) and the glycosyltransferase domain (between the 466 and 711 amino acids) (Fig. 3B). Amino acid 23 (Arg) was present before the exostosin and glycosyltransferase domains. Furthermore, evolutionary conservation analysis revealed that the amino acids were highly conservative in multiple species (Fig. 4). This result indicates that this amino acid serves a notable role in the function of the EXT2 gene and remains in the process of biological evolution. This nonsense mutation $23 \mathrm{R}>\mathrm{X}$ causes the EXT2 transcription process to be terminated prematurely and produces a truncated EXT2 protein, lacking the C-terminal 705 amino acids (Fig. 3B). Since the glycosyltransferase domain serves a notable role in HS biosynthesis, the mutation $23 \mathrm{R}>\mathrm{X}$ is likely to have a significant effect on the function of the EXT2 gene, particularly affecting the biosynthesis of HS. The same result was also observed with the online prediction tool Mutation Taster, which revealed that using stop codon to replace 23 arginine affects the function of the EXT2 protein and leads to the occurrence of the disease (probability score, 6.0) (Fig. 5).

\section{Discussion}

Although HMO is observed in Europe and the United States at an incidence of 1:50,000-100,000, to the best of our knowledge its incidence in China is not known $(3,4)$. Clinical characteristics often vary among individuals: Patients typically have symmetrical bony protrusions with cartilage caps in long bone growth plates, as well as short, flat and/or irregular bones; certain patients have disproportionate limbs and short stature (20). The onset age for half of all patients is $<3$ years old. During growth and development, exostoses grow gradually; the growth plates eventually disappear and exostoses stop growing until puberty (20). The clinical symptoms and complications of HMO are associated with the location and size of exostoses; these symptoms include pain, short stature, skeletal deformities, oppression around the blood vessels and nerves, 

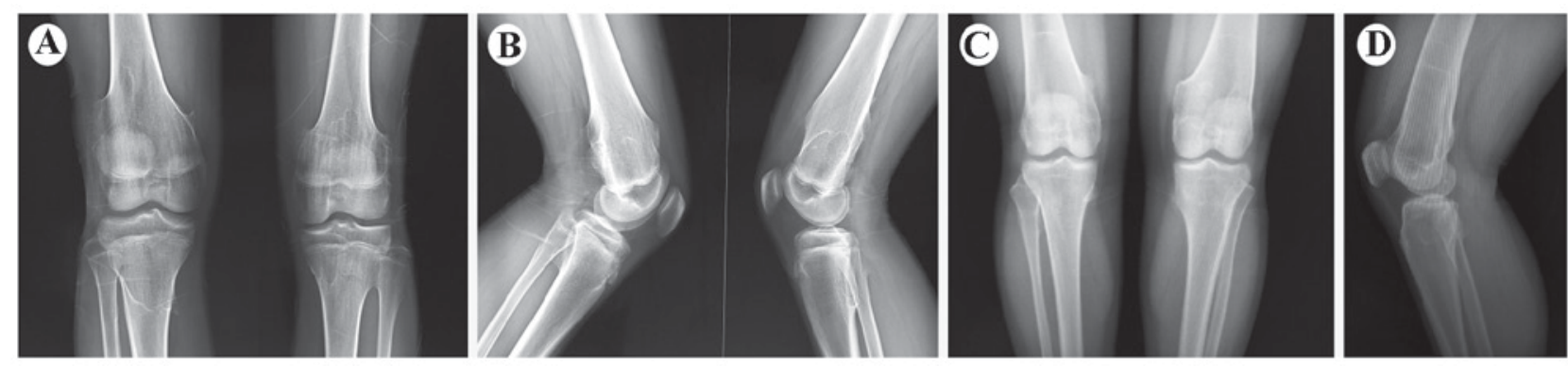

Figure 2. Radiograph images of patients, III1 (A and B) and II2 (C and D). (A-D) Multiple osteochondromas are observed around the bilateral knee.

A
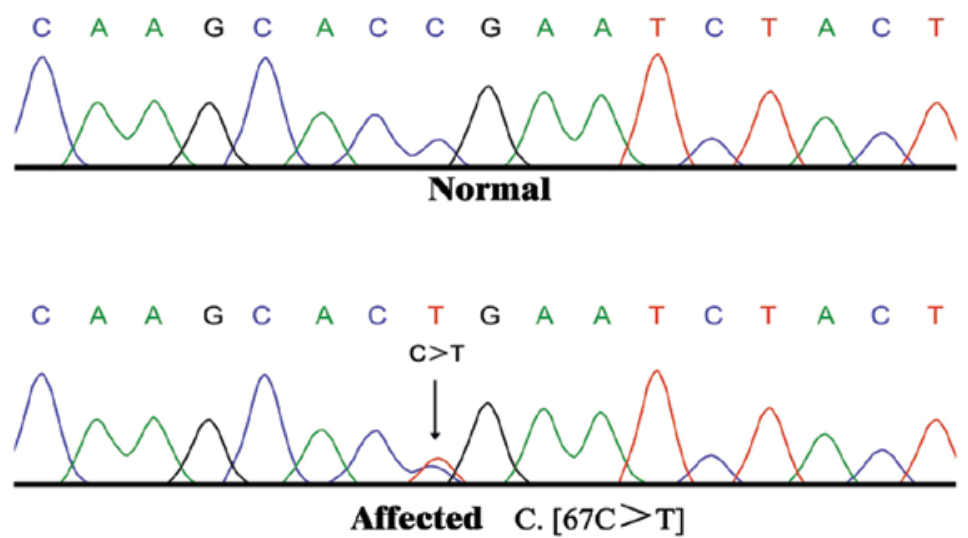

B

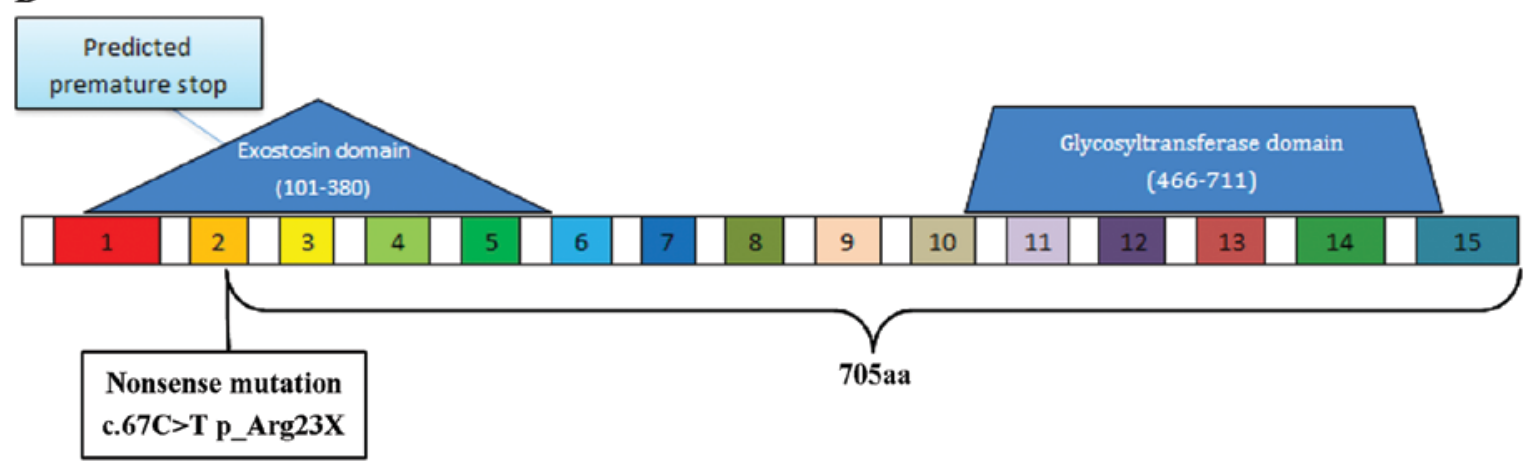

Figure 3. DNA sequencing was used to identify a novel mutation in the EXT2 gene. (A) The heterozygous nonsense mutation c.67C $>$ T (p.Arg $23 *$ ) was detected in the EXT2 gene of HMO patients (under panel) but not in that of healthy controls (upper panel). (B) The mutation c.(67C>T), located in exon 2, was predicted to introduce a premature stop codon at position 67, resulting in a truncation of 23 amino acids at the C-terminus of the EXT2 protein. EXT2, exostosin-2; HMO, hereditary multiple osteochondroma.

spontaneous pneumothorax and changes in joint mobility (4). The most serious complication is malignant chondrosarcoma or osteosarcoma, and the malignant transformation rate is $0.5-5 \%$ (12).

Human HMO is associated with at least two genes: EXT1 on 8q24.11-q24.13 and EXT2 on 11p12-11 $(21,22)$. These two tumor suppressor genes are widely expressed; EXT1 contains 11 exons and 10 introns and encodes 746 amino acids, whereas EXT2 contains 15 exons and 15 introns, with exons 2-14 form an open reading frame encoding 728 amino acids. The two genes are highly similar at the amino acid level, particularly in the $\mathrm{C}$-terminal region. According to Human Mutation Database statistics, 595 HMO-associated mutations have been detected to date, including 401 mutations in EXT1 and 194 mutations in EXT2; these include missense/nonsense mutations, small insertion/deletion mutations, splice site changes and large deletions (23). Although, to the best of our knowledge, the precise functions of these two genes is unknown, the proteins are localized in the type II transmembrane glycoproteins of the endoplasmic reticulum, which is associated with the synthesis of HS proteoglycan (HSPG) (17).

HSPG is composed of a core protein and an attached glycosaminoglycan chain (24). The proteins encoded by EXT1 and EXT2 form a heterologous oligomer in the Golgi apparatus of the majority of human cells (25). The heterologous oligomer, a glycosyltransferase complex, is involved in the polymerization of HS chains (26). The oligomer can add glucuronic acid and $\mathrm{N}$-acetylglucosamine residues to HS chains alternately, forming HSPGs (27). The activity of the glycosyltransferase complex formed by the EXT1/EXT2 oligomer is higher compared with that of EXT1 or EXT2 alone $(28,29)$. HS is widely expressed in cell membranes and the extracellular 


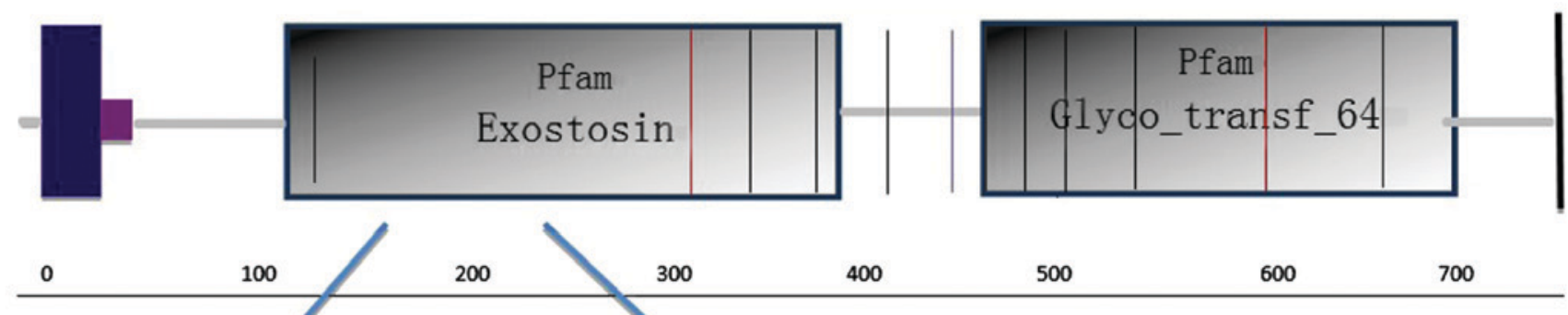

Exr2

Homo_sapiens_chromosome_11_clone_RP11-582N4

Homo_sapiens_exostosin_glycosyltransferase_2_(EXI2)

Hunañhereditary_multiple_exostoses_gene_2_(EXT2)_mRNA

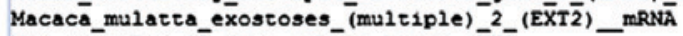

Mus_musculus_13

Mus_musculus_boñe_marrow_macrophağe_eDNA

Mus_musculus_exostoses_(multiple) 2-(Ext2) mRNA

Mus musculus exostoses (multiple) 2 mRNA (CDNA clone MGC 11478

Mus_musculus_multiple_exostoses_2_protein_(EXT2)_mRNA

otolemux_garñeti1__exostosin_2_(EXT2) _ ${ }^{m R N A}$

Consensus

Figure 4. Sequence alignment of EXT2 orthologs. EXT2, exostosin-2.
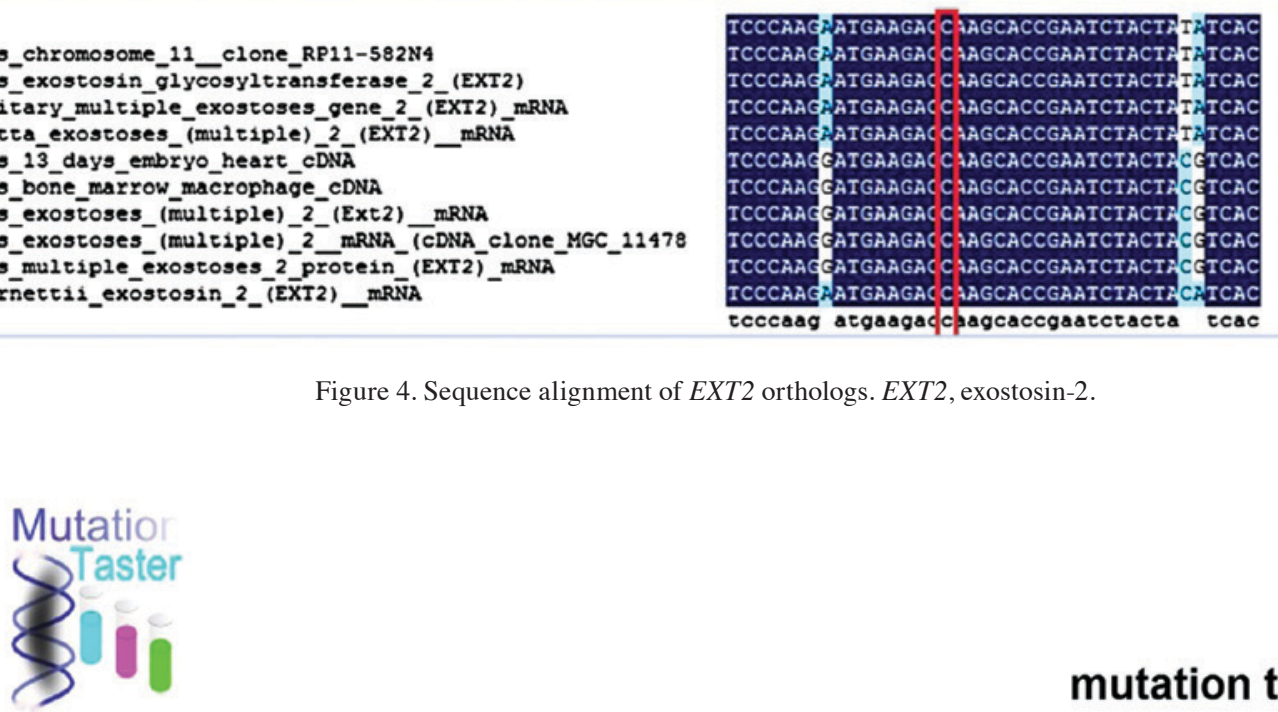

Prediction disease causing Model: complex_aae, prob: 1 (classification due to NMD, real probability is shown anyway) (explain)

\begin{tabular}{|c|c|}
\hline Summary & $\begin{array}{l}\text { - NMD } \\
\text { amino acid sequence changed } \\
\text { - known disease mutation at this position (HGMD CM980644) }\end{array}$ \\
\hline \multicolumn{2}{|c|}{ analysed issue analysis result } \\
\hline Name of alteration & no title \\
\hline $\begin{array}{l}\text { alteration (phys. } \\
\text { location) }\end{array}$ & chr11:44129329C>T \\
\hline HGNC symbol & EXT2 \\
\hline \multicolumn{2}{|c|}{$\begin{array}{l}\text { Ensembl transcript ENSTO0000358681 } \\
\text { ID }\end{array}$} \\
\hline UniProt peptide & Q93063 \\
\hline alteration type & single base exchange \\
\hline Alteration region & $\operatorname{CDS}$ \\
\hline DNA changes & $\begin{array}{l}\text { c. } 67 C>T \\
\text { CDNA.233C>T } \\
\text { g.12231C>T }\end{array}$ \\
\hline AA changes & R23* Score: 6.0 explain score(s) \\
\hline
\end{tabular}

Figure 5. MutationTaster predicted that the substitution of R23 to the termination codon causes diseases. EXT2, exostosin-2; NMD, nonsense-mediated mRNA decay.

matrix serves a notable role in receptor ligand binding, signal transduction and other physiological processes (30). HS proteoglycans are essential in several signaling pathways, including those involving fibroblast growth factors (FGFs), vascular endothelial growth factor and transforming growth factor- $\beta$, and may affect the concentration gradient of various morphogens on the cell surface, including bone morphogenetic proteins and hedgehog (31). HS proteoglycans can bind hedgehog ligands to control the spread of the hedgehog ligand in the extracellular matrix (32).

Hedgehog is a secretory protein that hydrolyzes itself, and the hedgehog signaling pathway serves a notable role in the regulation of growth plate chondrocytes (33-35). Hypertrophic chondrocytes of the growth plate may secrete Indian hedgehog (Ihh) $(36,37)$. Ihh functions with parathyroid hormone-related protein $(\mathrm{PTHrP})$ and regulates endochondral bone formation 
via a negative feedback loop (38). Ihh can induce periarticular hypertrophic chondrocytes to increase the secretion of PTHrP, which can suppress the proliferation of chondrocytes into hypertrophic chondrocytes (18). Ihh can also individually control chondrocyte differentiation and induce periosteal osteogenesis (39). In the regulation of endochondral bone growth via Ihh-PTHrP, multiple factors serve notable roles, including bone morphogenetic proteins (BMPs), which promote Ihh expression in cartilage cells (40).

Mutations in EXT1 and EXT2 can lead to the expression of truncated proteins that are associated with decreased activity of the glycosyltransferase complex (17). Synthesis of the HS chain is then reduced, which leads to the formation of incomplete HS proteoglycans $(26,27)$. The incomplete HS proteoglycans cause ligand-receptor binding disorders in a number of signaling pathways, including Ihh, BMPs, FGF, and Wnt, which affects the concentration gradient of ligands on the cell surface or in the extracellular matrix $(30,31)$. Disorders in the Ihh signaling pathway cause Ihh secretion and increase secretion of PTHrP by periarticular hypertrophic chondrocytes (18). This influences the proliferative potential of chondrocytes into hypertrophic chondrocytes and causes chondrocytes to undergo a long proliferative period, and ultimately HMO (41-44). Additionally, abnormal HS proteoglycans may change the morphology and differentiation potential of perichondrocytes, resulting in perichondrial cells that function as growth-plate-like cells and give rise to chondrocytes that clonally expand and develop into an exostosis, leading to HMO (25).

A lower disease burden is observed in individuals with $E X T 2$ mutations than in those with EXT1 mutations (15). In a study examining 172 individuals from 78 families, Porter et al (12) verified a higher severe disease frequency in persons with mutations in EXT1 than in those with EXT2 mutations, as evidenced by shortened stature, skeletal deformities (shortened forearm or bowing and knee deformities), and function (decreased range of motion in the elbow, forearm and/or knee) (13). Individuals with EXT2 mutations have fewer exostoses, a lower incidence of limb misalignment with longer limb segments and height, and less frequent pelvic and flat bone involvement than those with EXT1 mutations (45).

The present study reveals a novel heterozygous nonsense mutation (c.67C $>\mathrm{T}$ ) in EXT2, using whole-exome sequencing. The mutation was validated by Sanger sequencing and its negative influence on the expression of EXT2 was examined via in silico analysis. The results of the current study provide information regarding the association between the EXT gene family and HMO. These findings may facilitate early diagnosis and prenatal genetic screening of HMO.

\section{Acknowledgements}

The present study was supported by grants from the Natural Science Foundation of Zhejiang Province (grant no. Y2100731) and Zhejiang Medical Science and Technology Project (grant no. Y201024964).

\section{Competing interests}

The authors declare that they have no competing interests.

\section{References}

1. Black B, Dooley J, Pyper A and Reed M: Multiple hereditary exosloses. An epidemiologie study of an isolated community in Manitoba. Clin Oahop Ralat Res: 212-217, 1993.

2. Peterson HA: Multiple hereditary osteochondromata. Clin Orthop Relat Res: 222-230, 1989.

3. Hennekam RC: Hereditary multiple exostoses. J Med Genet 28: 262-266, 1991.

4. Schmale GA, Conrad EU III and Raskind WH: The natural history of hereditary multiple exostoses. J Bone Joint Surg Am 76: 986-992, 1994.

5. Legeai-Mallet L, Munnich A, Maroteaux P and Le Merrer M: Incomplete penetrance and expressivity skewing in hereditary multiple exostoses. Clin Genet 52: 12-16, 1997.

6. Wicklund CL, Pauli RM, Johnston D and Hecht JT: Natural history study of hereditary multiple exostoses. Am J Med Genet 55: 43-46, 1995.

7. Gordon SL, Buchanan JR and Ladda RL: Hereditarymultiple exostoses: Report of a kindred. J Med Genet 18: 428-430, 1981

8. Shupe JL, Leone NC, Olson AE and Gardner EJ: Hereditary multiple exostoses: Clinicopathologic features of a comparative study in horses and man. Am J Vet Res 40: 751-757, 1979.

9. Paik NJ, Han TR and Lim SJ: Multiple peripheral nerve compressions related to malignantly transformed hereditary multiple exostoses. Muscle Nerve 23: 1290-1294, 2000.

10. Khurana J, Abdul-Karim F and Bovée JVMG: Osteochondroma. In: Pathology and Genetics of Tumours of Soft Tissue and Bone. Fletehe CDM, Unni KK and Mertens F (eds). World Health Organization Classification of Tumours, Lyon, France, pp228230, 2002.

11. Saglik Y,Altay M,Unal VS, Basarir K and Yildiz Y: Manifestations and management of osteochondromas: A retrospective analysis of 382 patients. Acta Orthop Belg 72: 748-755, 2006.

12. Porter DE, Lonie L, Fraser M, Dobson-Stone C, Porter JR, Monaco AP and Simpson AH: Severity of disease and risk of malignant change in hereditary multiple exostoses. A genotype-phenotype study. J Bone Joint Surg Br 86: 1041-1046, 2004.

13. Stieber JR and Dormans JP: Manifestations of herecitary multiple exestoses. J Am Acac Orthop Surg 13: 110-120, 2005.

14. Francannet C, Cohen-Tanugi A, Le Merrer M, Munnich A, Bonaventure J and Legeai-Mallet L: Genotype-phenotype correlation in hereditary multiple exostoses. J Med Genet 38: 430-434, 2001.

15. Faiyaz-UI-Hague M, Ahmac W, Zaici SH, Hussain S, Haque S, Ahmad M, Cohn DH and Tsui LC: Novel mutations in the EXT1 gene in two consanguineous families affectec with multiple herecitary exostoses (familial osteochoncromatosis). Clin Genet 66: 144-151, 2004.

16. Kang Z, Peng F and Ling T: Mutation screening of EXT genes in Chinese patients with multiple osteochondromas. Gene 506: 298-300, 2012.

17. China National Physique Monitoring center. Workbook of public physique monitoring program, 2014 (In Chinese).

18. Schwarz JM, Rödelsperger C, Schuelke M and Seelow D: MutationTaster evaluates disease-causing potential of sequence alterations. Nat Methods 7: 575-576, 2010.

19. Cao L, Liu F, Kong M, Fang Y, Gu H, Chen Y, Zhao C, Zhang S and Bi Q: Novel EXT1 mutation identified in a pedigree with hereditary multiple exostoses. Oncol Rep 31: 713-718, 2014.

20. Bovée JV: Multiple osteochondromas. Orphanet J Rare Dis 3: 3: 2008.

21. Jennes I, Zuntini M, Mees K, Palagani A, Pedrini E, De Cock G, Fransen E, Vanden Berghe W, Sangiorgi L and Wuyts W: Identification and functional characterization of the human EXT1 promoter region. Gene 492: 148-159, 2012.

22. Wuyts W, Van Hul W, De Boulle K, Hendrickx J, Bakker E, Vanhoenacker F, Mollica F, Lüdecke HJ, Sayli BS, Pazzaglia UE, et al: Mutations in the EXT1 and EXT2 genes in hereditary multiple exostoses. Am J Hum Genet 62: 346-354, 1998.

23. Shimokawa K, Kimura-Yoshida C,Nagai N,Mukai K, MatsubaraK, Watanabe H, Matsuda Y, Mochida K and Matsuo I: Cell surface heparan sulfate chains regulate local reception of FGF signaling in the mouse embryo. Dev Cell 21: 257-272, 2011.

24. Yanagishita M and Hascall VC: Cell surface heparan sulfate proteoglycans. J Biol Chem 267: 9451-9454, 1992.

25. Hecht JT, Hayes E, Haynes R, Cole WG, Long RJ, Farach-Carson MC and Carson DD: Differentiation-induced loss of heparan sulfate in human exostosis derived chondrocytes. Differentiation 73: 212-221, 2005. 
26. Lindahl U: Heparan sulfate-protein interactions-a concept for drug design. Thromb Haemost 98: 109-115, 2007.

27. Kim BT, Kitagawa H, Tamura J, Saito T, Kusche-Gullberg M, Lindahl U and Sugahara K: Human tumor suppressor EXT gene family members EXTL1 and EXTL3 encode alpha 1,4- N-acetylglucosaminyltransferases that likely are involved in heparan sulfate/ heparin biosynthesis. Proc Natl Acad Sci USA 98: 7176-7181, 2001.

28. Busse M, Feta A, Presto J, Wilén M, Grønning M, Kjellén L and Kusche-Gullberg M: Contribution of EXT1, EXT2, and EXTL3 to heparan sulfate chain elongation. J Biol Chem 282: 32802-32810, 2007.

29. Mccormick C, Duncan G, Goutsos KT and Tufaro F: The putative tumor suppressors EXT1 and EXT2 form a stable complex that accumulates in the Golgi apparatus and catalyzes the synthesis of heparan sulfate. Proc Natl Acad Sci USA 97: 668-673, 2000.

30. Esko JD and Selleck SB: Order out of chaos: Assembly of ligand binding sites in heparan sulfate. Annu Rev Biochem 71: 435-471, 2002.

31. Nadanaka $\mathrm{S}$ and Kitagawa $\mathrm{H}$ : Heparan sulphate biosynthesis and disease. J Biochem 144: 7-14, 2008.

32. The I, Bellaiche Y and Perrimon N: Hedgehog movement is regulated through tout velu-dependent synthesis of a heparan sulfate proteoglycan. Mol Cell 4: 633-639, 1999.

33. Maeda Y, Nakamura E, Nguyen MT, Suva LJ, Swain FL, Razzaque MS, Mackem S and Lanske B: Indian Hedgehog produced by postnatal chondrocytes is essential for maintaining a growth plate and trabecular bone. Proc Natl Acad Sci USA 104: 6382-6387, 2007.

34. Wang W, Lian N, Li L, Moss HE, Wang W, Perrien DS, Elefteriou $F$ and Yang X: Atf4 regulates chondrocyte proliferation and differentiation during endochondral ossification by activating Ihh transcription. Development 136: 4143-4153, 2009.

35. Lin AC, Seeto BL, Bartoszko JM, Khoury MA, Whetstone H, Ho L, Hsu C, Ali SA and Alman BA: Modulating hedgehog signaling can attenuate the severity of osteoarthritis. Nat Med 15 $1421-1425,2009$
36. Pathi S, Rutenberg JB, Johnson RL and Vortkamp A: Interaction of Ihh and BMP/Noggin signaling during cartilage differentiation. Dev Biol 209: 239-253, 1999.

37. Bitgood MJ and Mcmahon AP: Hedgehog and Bmp genes are coexpressed at many diverse sites of cell-cell interaction in the mouse embryo. Dev Biol 172: 126-138, 1995.

38. Kobayashi T, Lyons KM, McMahon AP and Kronenberg HM: BMP signaling stimulates cellular differentiation at multiple steps during cartilage development. Proc Natl Acad Sci USA 102: 18023-18027, 2005.

39. Kronenberg HM: Developmental regulation of the growth plate. Nature 423: 332-336, 2003.

40. Minina E, Kreschel C, Naski MC, Ornitz DM and Vortkamp A: Interaction of FGF, Ihh/Pthlh, and BMP signaling integrates chondrocyte proliferation and hypertrophic differentiation. Dev Cell 3: 439-449, 2002.

41. Stickens D, Clines G, Burbee D, Ramos P, Thomas S, Hogue D, Hecht JT, Lovett M and Evans GA: The EXT2 multiple exostoses gene defines a family of putative tumour suppressor genes. Nat Genet 14: 25-32, 1996.

42. Bornemann DJ, Park S, Phin S and Warrior R: A translational block to HSPG synthesis permits BMP signaling in the early Drosophila embryo. Development 135: 1039-1047, 2008.

43. Bornemann DJ, Duncan JE, Staatz W, Selleck S and Warrior R: Abrogation of heparan sulfate synthesis in Drosophila disrupts the Wingless, Hedgehog and Decapentaplegic signaling pathways. Development 131: 1927-1938, 2004.

44. Bishop JR, Schuksz M and Esko JD: Heparan sulphate proteoglycans fine-tune mammalian physiology. Nature 446: 1030-1037, 2007.

45. Alvarez C, Tredwell S, De Vera M and Hayden M: The genotype-phenotype correlation of hereditary multiple exostoses. Clin Genet 70: 122-130, 2006.

This work is licensed under a Creative Commons Attribution-NonCommercial-NoDerivatives 4.0 International (CC BY-NC-ND 4.0) License. 